\title{
Existence-uniqueness for Stochastic Functional Differential Equations with Non-Lipschitz Coefficients
}

\author{
Lingying Teng ${ }^{1 *}$ and Xiaohu Wang ${ }^{2}$ \\ ${ }^{1}$ College of Computer science and Technology of Southwest University for Nationalities, Chengdu, Sichuan, \\ China \\ ${ }^{2}$ Yangtze Center of Mathematics, Sichuan University, Chengdu, Sichuan, China \\ Email: tly82@126.com
}

\begin{abstract}
The main aim of this paper is to develop some basic theories of stochastic functional differential equations (SFDEs) with non-Lipschitz coefficients. Firstly, we show that Peano's theorem holds for SFDEs, that is, the continuity alone is sufficient to prove the local existence of the initial value problem of SFDEs. Secondly, some new uniqueness theorems are established by the comparison methods proposed by $\mathrm{Xu}$ et al. And then, continuation theorems and global existence theorems for SFDEs with non-Lipschitz coefficients are obtained. Finally, an example is given to illustrate the efficiency of the obtained results.
\end{abstract}

Keywords: Stochastic functional differential equations, non-Lipschitz, existence, uniqueness, continuation theorem.

\section{Introduction}

Many physical phenomena can be modelled by stochastic dynamical systems whose evolution on time is governed by random forces as well as intrinsic dependence of the state on a finite part of its past history. Such models may be identified as SFDEs. In recent years, the investigation for SFDEs has attracted the considerable attention of researchers and many qualitative properties of solutions to SFDEs have been obtained. Some important results can be found in $[1,2,3]$ and references cited therein. It is well known that most of the existence and uniqueness theorems for SFDEs were established under Lipschitz conditions. The important representative works are as follows.

Mao [3] considered the following SFDE of Itô-type

$$
\mathrm{d} x(t)=f\left(t, x_{t}\right) \mathrm{d} t+g\left(t, x_{t}\right) \mathrm{d} \omega(t), \quad t_{0} \leq t<T,
$$

with the initial condition $x_{t_{0}}=\xi$, where $x_{t}(s)=x(t+s), s \in[-\tau, 0], \tau>0, T$ is a constant, or $T=\infty$.

Mao obtained the existence-uniqueness of solutions of the initial value problem (1) if $f$ and $g$ satisfy linear growth condition and uniform Lipschitz condition or quasi-local Lipschitz condition [3, Theorem 2.2 and Theorem 2.5]. Xu et al. [4, Lemma 3.1] obtained the existence-uniqueness of solutions for SFDE (1) if $f$ and $g$ only satisfy uniform Lipschitz condition. In [5], Xu et al. presented the existence-uniqueness theorems of solutions for SFDE (1) if $f$ and $g$ are continuous and satisfy local Lipschitz condition. By means of the successive approximations method, Turo [6] investigated the local or global existence and uniqueness of solutions to SFDE (1) under non-Lipschitz condition. Ren et al. [7] obtained the existenceuniqueness of solutions for SFDEs with infinite delay under non-Lipschitz condition and a weakened linear growth condition. Base on the Razumikhin technique and compare principle, Xu et al.[8] obtained the moment estimate and existence of solutions for SFDEs with discontinuous initial functions.

It is well known that Peano's theorem is a classical and fundamental existence theorem for the initial value problem of ordinary differantial equations (ODEs)[9]. Peano's theorem shows that continuity alone is sufficient to prove a local existence theorem for ODEs. Up to now, Peano's theorem has been extended to many cases such as ellipitic differential equations [10], functional differential equations [11], differential inclusions [12], fuzzy differential equations [13,14,15], fractional differential equations [16]. However, the Peano's theorem for SFDE (1) has not yet been established prior to this work and it is still open.

Motivated by the discussions above, a natural question then is that of asking whether Peano's theorem holds for SFDE (1)? That is, is the continuity alone sufficient to prove a local existence theorem for SFDE 
(1)? One of our main objectives is to give a positive answer of the above question. Our new theorems need only the continuity condition but neither the Lipschitz condition nor the non-Lipschitz condition used in [6,7], therefore the theorems cover a wider class of nonlinear SFDEs. But, our existence theorems can not guarantee the uniqueness of the solutions. Therefore, our second objective is to establish some general uniqueness theorems. Finally, we will give some continuation and global existence theorems for SFDE (1) [5] under non-Lipschitz condition with Lipschitz condition being considered as a special case.

This paper is organized as follows. In section 2, we introduce some notations and definitions. Section 3 is devoted to obtain the Peano's theorem for SFDE (1). In section 4, we will establish some new uniqueness theorems for SFDE (1). In section 5, we shall give some continuation theorems and global existence theorems for SFDE (1). An example is given in section 6 to illustrate the efficiency of the obtained results.

\section{Preliminaries}

In this section, we introduce some notations and recall some basic definitions.

$C(X, Y)$ denotes the space of continuous mappings from the topological space $X$ to the topological space $Y$. Especially, let $C \triangleq C\left([-\tau, 0], R^{n}\right)$ with the norm $\|\varphi\|=\sup _{-\tau \leq s \leq 0}|\varphi(s)|$, where $\tau>0$ and $|\cdot|$ is any norm in $R^{n}$.

Let $\left(\Omega, \mathcal{F},\left\{\mathcal{F}_{t}\right\}_{t \geq t_{0}}, P\right)$ be a complete probability space with a filtration $\left\{\mathcal{F}_{t}\right\}_{t \geq t_{0}}$ satisfying the usual conditions ( i.e. it is right continuous and $\mathcal{F}_{t_{0}}$ contains all $P$-null sets in $\left.\mathcal{F}\right) . \omega(t)=\left(\omega_{1}(t), \ldots, \omega_{m}(t)\right)^{T}$ is an $m$-dimensional Brownian motion defined on $\left(\Omega, \mathcal{F},\left\{\mathcal{F}_{t}\right\}_{t \geq t_{0}}, P\right)$.

For Banach space $L^{p}\left(\Omega, R^{n}\right), p>0$, we define the norm

$$
|x|_{\Omega} \triangleq\left(\mathbb{E}|x|^{p}\right)^{\frac{1}{p}}, \quad p>0
$$

We also employ $|\cdot|_{\Omega}$ to denote the norm of Banach space $L^{p}\left(\Omega, R^{n \times m}\right), p>0$.

We shall adopt the usual manner (see $[3,5])$ and let $L^{p}\left(\Omega, C\left(J, R^{n}\right)\right), p>0$, be the space of $(\mathcal{F}$, Borel C)-measurable maps $\Omega \rightarrow C\left(J, R^{n}\right)$ which are $L^{p}$ in the Bochner sense. Give $L^{p}\left(\Omega, C\left(J, R^{n}\right)\right)$ the norm

$$
\|\xi\|_{\Omega J}=\left[\int_{\Omega} \sup _{t \in J}|\xi(t, \omega)|^{p} \mathrm{~d} P(\omega)\right]^{\frac{1}{p}}=\left[\mathbb{E} \sup _{t \in J}|\xi(t, \omega)|^{p}\right]^{\frac{1}{p}}, \quad p>0,
$$

where $J \subset R$ is a bounded interval. Especially, when $J=[-\tau, 0]$,

$$
L^{p}\left(\Omega, C\left(J, R^{n}\right)\right)=L^{p}(\Omega, C) .
$$

For convenience, we denote the norm of $\xi \in L^{p}(\Omega, C)$ by

$$
\|\xi\|_{\Omega} \triangleq\|\xi\|_{\Omega[-\tau, 0]}=\left[\mathbb{E}\|\xi\|^{p}\right]^{\frac{1}{p}}, \quad p>0 .
$$

Let $L_{D}^{p}\left(\Omega, C\left(\left[t_{0}-\tau, a\right], R^{n}\right)\right)$ be the space of all processes $x(t) \in L^{p}\left(\Omega, C\left(\left[t_{0}-\tau, a\right], R^{n}\right)\right)$ such that $x(t)$ is $\mathcal{F}_{t_{0}}$-measurable for all $t \in\left[t_{0}-\tau, t_{0}\right]$ and $x(t)$ is $\mathcal{F}_{t}$-measurable for all $t \in\left[t_{0}, a\right]$. Then, $L_{D}^{p}\left(\Omega, C\left(\left[t_{0}-\tau, a\right], R^{n}\right)\right)$ is a closed linear subspace of $L^{p}\left(\Omega, C\left(\left[t_{0}-\tau, a\right], R^{n}\right)\right)[2$, P. 31].

Throughout this paper, we always set $p \geq 2$ and suppose that $\xi \in L^{p}(\Omega, C)$ is an $\mathcal{F}_{t_{0}}$-measurable process and for (1), the drift coefficient function $f:\left[t_{0}, T\right) \times L^{p}(\Omega, C) \rightarrow L^{p}\left(\Omega, R^{n}\right)$ and the diffusion coefficient $g:\left[t_{0}, T\right) \times L^{p}(\Omega, C) \rightarrow L^{p}\left(\Omega, R^{n \times m}\right)$ are both Borel measurable.

Definition 2.1. Let $\bar{J}=\left[t_{0}-\tau, a\right)$ or $\bar{J}=\left[t_{0}-\tau, a\right]$, where $t_{0}<a \leq T$. $R^{n}$-value stochastic process $x(t)$ defined on $\bar{J}$ is called a solution of (1) if $x(t) \in L_{D}^{p}\left(\Omega, C\left(\bar{J}, R^{n}\right)\right)$ and satisfies (1) almost surely. The solution $x(t)$ of (1) on interval $\bar{J}$ is said to be unique if any other solution $\bar{x}(t)$ on interval $\bar{J}$ is indistinguishable from it, that is

$$
P\{x(t)=\bar{x}(t) \text { for all } t \in \bar{J}\}=1 \text {. }
$$


Definition 2.2. Let $x(t)$ on $J_{1}$ and $\bar{x}(t)$ on $J_{2}$ both be solutions of (1). If $J_{1} \subset J_{2}, J_{1} \neq J_{2}$ and $P\left\{x(t)=\bar{x}(t)\right.$ for all $\left.t \in J_{1}\right\}=1$, we say $\bar{x}(t)$ is a continuation of $x(t)$, or $x(t)$ can be continued to $J_{2}$. A solution $x(t)$ is non-continuable if it has no continuation. The existing interval of non-continuable solution $x(t)$ is called the maximum existing interval of $x(t)$.

Definition 2.3. The functional $F:\left[t_{0}, T\right) \times L^{p}(\Omega, C) \rightarrow L^{p}\left(\Omega, R^{n}\right)$ is said to be quasi-bounded if for any constants $\beta \in\left(t_{0}, T\right)$ and $\alpha>0$, there exists a positive constant $M$ such that

$$
|F(t, \phi)|_{\Omega} \leq M
$$

provided that

$$
t \in\left[t_{0}, \beta\right] \text { and }\|\phi\|_{\Omega} \leq \alpha .
$$

Definition 2.4. It is said that a sample path $x(t, \omega)$ explodes in $\left[t_{0}-\tau, T\right)$ if for any integer $k>0$, there exists a time $s \in\left[t_{0}-\tau, T\right)$ such that $|x(s, \omega)| \geq k$. And the solution $x(t, \omega)$ of $(1)$ explodes in $\left[t_{0}-\tau, T\right)$ if there exists a measurable subset $S \subset \Omega$ with $P(S)>0$ such that the sample path $x(t, \omega)$ explodes in $\left[t_{0}-\tau, T\right)$ for almost all $\omega \in S$.

Definition 2.5. A function $h \in C\left(R \times R^{m} \times R^{m}, R^{m}\right)$ is called an $H_{m}$-function if for any $t \geq t_{0} \in R$ and any $u^{(1)}, u^{(2)}, v^{(1)}, v^{(2)} \in R^{m}$, every ith element of $h$ satisfies $h_{i}\left(t, u^{(1)}, v^{(1)}\right) \leq h_{i}\left(t, u^{(2)}, v^{(2)}\right)$ when $u^{(1)} \leq u^{(2)}$ with $u_{i}^{(1)}=u_{i}^{(2)}$ and $v^{(1)} \leq v^{(2)}$.

\section{Local Existence Theorem}

In this section, we give a local existence theorem for SFDE (1) assuming that $f$ and $g$ are only continuous.

Lemma 3.1. Set $p \geq 2$. For any $t_{0} \in R$ and $\beta>0$, let $g$ be a process in $L^{p}\left(\left[t_{0}, t_{0}+\beta\right] ; R^{n \times m}\right)$ such that $\mathbb{E} \int_{t_{0}}^{t_{0}+\beta}|g(s)|^{p} \mathrm{~d} s<\infty$, then

$$
\mathbb{E}\left|\int_{t_{0}}^{t_{0}+\beta} g(s) \mathrm{d} \omega(s)\right|^{p} \leq C_{p} \beta^{\frac{p-2}{2}} \mathbb{E} \int_{t_{0}}^{t_{0}+\beta}|g(s)|^{p} \mathrm{~d} s, \text { where } C_{p}=\left(\frac{p(p-1)}{2}\right)^{\frac{p}{2}} .
$$

Proof. The proof is similar to that of Theoerm 7.1 in [3] except letting

$$
x(t)=\int_{t_{0}}^{t} g(s) \mathrm{d} \omega(s), \quad \forall t \in\left[t_{0}, t_{0}+\beta\right],
$$

and noting $x\left(t_{0}\right)=0$. So it is omitted.

Definition 3.1. Let $Y$ be a subset of a Banach space $X$ and $f: Y \rightarrow X$. If $f$ is continuous and $f(Y)$ is contained in a compact subset of $X$, then $f$ is a compact mapping.

Lemma 3.2. (Schauder fixed-point theorem, see in [17, p.184]) Let $Y$ be a nonempty convex subset of a Banach space $X$ and $\Gamma: Y \rightarrow Y$ be a compact mapping. Then $\Gamma$ has a fixed point.

Theorem 3.1. (Local existence theorem) Assume $f$ and $g$ are continuous on $\left[t_{0}, T\right) \times L^{p}(\Omega, C)$. Then there is a $\beta>0$ such that the SFDE (1) has a solution on $\left[t_{0}-\tau, t_{0}+\beta\right]$.

Proof. Since $f$ and $g$ are continuous, for the above given $\xi$ and any $x_{t} \in L^{p}(\Omega, C)$, there exists a positive constant $\delta \leq 1$ such that if $\left|t-t_{0}\right| \leq \delta$ and $\left\|x_{t}-\xi\right\|_{\Omega} \leq \delta$, then

$$
\left|f\left(t, x_{t}\right)-f\left(t_{0}, \xi\right)\right|_{\Omega} \leq 1, \quad\left|g\left(t, x_{t}\right)-g\left(t_{0}, \xi\right)\right|_{\Omega} \leq 1
$$

Therefore, for $\left|t-t_{0}\right| \leq \delta$ and $\left\|x_{t}-\xi\right\|_{\Omega} \leq \delta$,

$$
\left|f\left(t, x_{t}\right)\right|_{\Omega} \vee\left|g\left(t, x_{t}\right)\right|_{\Omega} \leq\left[\left|f\left(t_{0}, \xi\right)\right|_{\Omega} \vee\left|g\left(t_{0}, \xi\right)\right|_{\Omega}\right]+1 \triangleq M
$$


Since $\xi \in L^{p}(\Omega, C)$, then for the above $\delta$, there is a $\delta_{0}>0$ such that

$$
|\xi(s+r)-\xi(s)|_{\Omega} \leq \frac{\delta}{2}, \quad \forall|r| \leq \delta_{0}
$$

For $\alpha>|\xi(0)|_{\Omega}$, we choose $\beta=\min \left\{1, \delta_{0},\left[\frac{\delta}{2 M\left(1+C_{p}^{\frac{1}{p}}\right)}\right]^{2},\left[\frac{\alpha-|\xi(0)|_{\Omega}}{M\left(1+C_{p}^{\frac{1}{p}}\right)}\right]^{2}\right\}$ and denote

$$
\begin{aligned}
S(\alpha, \beta)= & \left\{x \in L^{p}\left(\Omega, C\left(\left[t_{0}-\tau, t_{0}+\beta\right], R^{n}\right)\right):|x(t)|_{\Omega} \leq \alpha \text { on }\left[t_{0}-\tau, t_{0}+\beta\right] ;\right. \\
& \left|x\left(t_{1}\right)-x\left(t_{2}\right)\right|_{\Omega} \leq M\left(1+C_{p}^{\frac{1}{p}}\right)\left|t_{1}-t_{2}\right|^{\frac{1}{2}} \text { on }\left[t_{0}, t_{0}+\beta\right] ; x(t)=\xi\left(t-t_{0}\right) \\
& \text { for } \left.t_{0}-\tau \leq t \leq t_{0}\right\},
\end{aligned}
$$

where $C_{p}$ is defined in Lemma 3.1. The set $S(\alpha, \beta)$ is convex and contained in a Banach space. Let

$$
\Gamma(x)(t)=\left\{\begin{array}{l}
\xi(0)+\int_{t_{0}}^{t} f\left(s, x_{s}\right) \mathrm{d} s+\int_{t_{0}}^{t} g\left(s, x_{s}\right) \mathrm{d} \omega(s), \quad t \geq t_{0}, \\
\xi\left(t-t_{0}\right), \quad t \in\left[t_{0}-\tau, t_{0}\right] .
\end{array}\right.
$$

Then $\Gamma$ is clearly continuous.

Now, we will show

$$
\Gamma: S(\alpha, \beta) \rightarrow S(\alpha, \beta), \quad \forall t \in\left[t_{0}, t_{0}+\beta\right] .
$$

Let $s \in[-\tau, 0]$ and $v \in\left[t_{0}, t_{0}+\beta\right]$. If $s+v \leq t_{0}$, by the definition in (5), we have

$$
|x(s+v)-\xi(s)|_{\Omega}=\left|\xi\left(s+v-t_{0}\right)-\xi(s)\right|_{\Omega} \leq \frac{\delta}{2}
$$

since $0 \leq v-t_{0} \leq \beta \leq \delta_{0}$.

If $s+v \geq t_{0}$, then,

$$
\begin{aligned}
|x(s+v)-\xi(s)|_{\Omega} & \leq|x(s+v)-\xi(0)|_{\Omega}+|\xi(0)-\xi(s)|_{\Omega} \\
& \leq|x(s+v)-\xi(0)|_{\Omega}+\frac{\delta}{2} \\
& \leq\left|x(s+v)-x\left(t_{0}\right)\right|_{\Omega}+\frac{\delta}{2} \\
& \leq M\left(1+C_{p}^{\frac{1}{p}}\right) \beta^{\frac{1}{2}}+\frac{\delta}{2} \leq \delta
\end{aligned}
$$

because $0 \leq-s \leq v-t_{0} \leq \beta \leq \delta_{0}$.

The above expression means that for any $v \in\left[t_{0}, t_{0}+\beta\right], s \in[-\tau, 0]$,

$$
|x(v+s)-\xi(s)|_{\Omega}=\left[\mathbb{E}|x(v+s)-\xi(s)|^{p}\right]^{\frac{1}{p}} \leq \delta .
$$

Thus, we conclude that for any $v \in\left[t_{0}, t_{0}+\beta\right]$,

$$
\left\|x_{v}-\xi\right\|_{\Omega}=\left[\mathbb{E} \sup _{-\tau \leq s \leq 0}|x(v+s)-\xi(s)|^{p}\right]^{\frac{1}{p}} \leq \delta .
$$

Therefore, the inequality (3) holds.

On the other hand, for any $t \in\left[t_{0}, t_{0}+\beta\right]$,

$$
|\Gamma(x)(t)|_{\Omega} \leq|\xi(0)|_{\Omega}+\left|\int_{t_{0}}^{t} f\left(s, x_{s}\right) \mathrm{d} s\right|_{\Omega}+\left|\int_{t_{0}}^{t} g\left(s, x_{s}\right) \mathrm{d} \omega(s)\right|_{\Omega} .
$$

By Hölder's inequality and (3), we have

$$
\left|\int_{t_{0}}^{t} f\left(s, x_{s}\right) \mathrm{d} s\right|_{\Omega}^{p}=\mathbb{E}\left|\int_{t_{0}}^{t} f\left(s, x_{s}\right) \mathrm{d} s\right|^{p}
$$




$$
\begin{aligned}
& \leq \beta^{p-1} \int_{t_{0}}^{t} \mathbb{E}\left|f\left(s, x_{s}\right)\right|^{p} \mathrm{~d} s \\
& \leq \beta^{p-1} \int_{t_{0}}^{t}\left|f\left(s, x_{s}\right)\right|_{\Omega}^{p} \mathrm{~d} s \\
& \leq M^{p} \beta^{p} .
\end{aligned}
$$

From Lemma 3.1, we get

$$
\begin{aligned}
\left|\int_{t_{0}}^{t} g\left(s, x_{s}\right) \mathrm{d} \omega(s)\right|_{\Omega}^{p} & =\mathbb{E}\left|\int_{t_{0}}^{t} g\left(s, x_{s}\right) \mathrm{d} \omega(s)\right|^{p} \\
& \leq C_{p}\left(t-t_{0}\right)^{\frac{p-2}{2}} \mathbb{E} \int_{t_{0}}^{t}\left|g\left(s, x_{s}\right)\right|^{p} \mathrm{~d} s \\
& \leq C_{p} \beta^{\frac{p-2}{2}} \int_{t_{0}}^{t} \mathbb{E}\left|g\left(s, x_{s}\right)\right|^{p} \mathrm{~d} s \\
& =C_{p} \beta^{\frac{p-2}{2}} \int_{t_{0}}^{t}\left|g\left(s, x_{s}\right)\right|_{\Omega}^{p} \mathrm{~d} s \\
& \leq C_{p} \beta^{\frac{p}{2}} M^{p}
\end{aligned}
$$

Then, by the definition of $\beta$, we obtain

$$
|\Gamma(x)(t)|_{\Omega} \leq|\xi(0)|_{\Omega}+M \beta+C_{p}^{\frac{1}{p}} \beta^{\frac{1}{2}} M \leq|\xi(0)|_{\Omega}+M\left(1+C_{p}^{\frac{1}{p}}\right) \beta^{\frac{1}{2}} \leq \alpha .
$$

To show that $\Gamma S(\alpha, \beta) \subseteq S(\alpha, \beta)$, for $t^{\prime}, t^{\prime \prime} \in\left[t_{0}, t_{0}+\beta\right]$, we shall estimate

$$
\begin{aligned}
\left|\Gamma(x)\left(t^{\prime}\right)-\Gamma(x)\left(t^{\prime \prime}\right)\right|_{\Omega} & =\left|\int_{t^{\prime \prime}}^{t^{\prime}} f\left(s, x_{s}\right) \mathrm{d} s+\int_{t^{\prime \prime}}^{t^{\prime}} g\left(s, x_{s}\right) \mathrm{d} \omega(s)\right|_{\Omega} \\
& \leq\left|\int_{t^{\prime \prime}}^{t^{\prime}} f\left(s, x_{s}\right) \mathrm{d} s\right|_{\Omega}+\left|\int_{t^{\prime \prime}}^{t^{\prime}} g\left(s, x_{s}\right) \mathrm{d} \omega(s)\right|_{\Omega} .
\end{aligned}
$$

By the same way in (12) and (13), we have

$$
\begin{aligned}
\left|\int_{t^{\prime \prime}}^{t^{\prime}} f\left(s, x_{s}\right) \mathrm{d} s\right|_{\Omega}^{p} & =\mathbb{E}\left|\int_{t^{\prime \prime}}^{t^{\prime}} f\left(s, x_{s}\right) \mathrm{d} s\right|^{p} \\
& \leq\left|t^{\prime}-t^{\prime \prime}\right|^{p-1} \int_{t^{\prime \prime}}^{t^{\prime}} \mathbb{E}\left|f\left(s, x_{s}\right)\right|^{p} \mathrm{~d} s \\
& \leq\left|t^{\prime}-t^{\prime \prime}\right|^{p-1} \int_{t^{\prime \prime}}^{t^{\prime}}\left|f\left(s, x_{s}\right)\right|_{\Omega}^{p} \mathrm{~d} s \\
& \leq M^{p}\left|t^{\prime}-t^{\prime \prime}\right|^{p}
\end{aligned}
$$

and

$$
\begin{aligned}
\left|\int_{t^{\prime \prime}}^{t^{\prime}} g\left(s, x_{s}\right) \mathrm{d} \omega(s)\right|_{\Omega}^{p} & =\mathbb{E}\left|\int_{t^{\prime \prime}}^{t^{\prime}} g\left(s, x_{s}\right) \mathrm{d} \omega(s)\right|^{p} \\
& \leq C_{p}\left|t^{\prime}-t^{\prime \prime}\right|^{\frac{p-2}{2}} \mathbb{E} \int_{t^{\prime \prime}}^{t^{\prime}}\left|g\left(s, x_{s}\right)\right|^{p} \mathrm{~d} s \\
& \leq C_{p}\left|t^{\prime}-t^{\prime \prime}\right|^{\frac{p-2}{2}} \int_{t^{\prime \prime}}^{t^{\prime}} \mathbb{E}\left|g\left(s, x_{s}\right)\right|^{p} \mathrm{~d} s \\
& =C_{p}\left|t^{\prime}-t^{\prime \prime}\right|^{\frac{p-2}{2}} \int_{t^{\prime \prime}}^{t^{\prime}}\left|g\left(s, x_{s}\right)\right|_{\Omega}^{p} \mathrm{~d} s \\
& \leq C_{p}\left|t^{\prime}-t^{\prime \prime}\right|^{\frac{p}{2}} M^{p}
\end{aligned}
$$


Then, we get

$$
\begin{aligned}
\left|\Gamma(x)\left(t^{\prime}\right)-\Gamma(x)\left(t^{\prime \prime}\right)\right|_{\Omega} & \leq M\left|t^{\prime}-t^{\prime \prime}\right|+M C_{p}^{\frac{1}{p}}\left|t^{\prime}-t^{\prime \prime}\right|^{\frac{1}{2}} \\
& \leq M\left(1+C_{p}^{\frac{1}{p}}\right)\left|t^{\prime}-t^{\prime \prime}\right|^{\frac{1}{2}} .
\end{aligned}
$$

From (13) and (16), we obtain $\Gamma S(\alpha, \beta) \subseteq S(\alpha, \beta)$. Moreover, from the definition of $S(\alpha, \beta)$ and ArzelàAscoli theorem [18, p.772], $S(\alpha, \beta)$ is compact. Then, by Lemma 3.2, there is a fixed point $x(t)$ which, as it is easy to see, is a solution of (1) with initial data $x_{t_{0}}=\xi$. The proof is completed.

Remark 3.1. Theorem 3.1 is a natural generalization of the local existence theorem [11,17] of the functional differential equation

$$
\dot{x}(t)=f\left(t, x_{t}\right), \quad x_{t_{0}}=\xi \in C,
$$

where $x \in R^{n}$ and $f \in C\left(\left[t_{0},+\infty\right) \times C, R^{n}\right)$.

\section{Local Uniqueness Theorems}

In this section, we will establish some uniqueness theorems of solutions of SFDE (1) by using comparison theorems established in [5].

For the vector functions $x(t)=\left(x_{1}(t), \ldots, x_{m}(t)\right)^{T} \in C\left(R, R^{m}\right)$, we denote

$$
\bar{x}(t)=\left(\bar{x}_{1}(t), \ldots, \bar{x}_{m}(t)\right)^{T}, \bar{x}_{i}(t)=\sup _{-\tau \leq s \leq 0} x_{i}(t+s), i=1,2, \ldots, m,
$$

and define the Dini upper right derivative as follows:

$$
D^{+} x(t)=\left(D^{+} x_{1}(t), \ldots, D^{+} x_{m}(t)\right)^{T}, \quad D^{+} x_{i}(t)=\limsup _{h \rightarrow 0^{+}} \frac{x_{i}(t+h)-x_{i}(t)}{h} .
$$

Lemma 4.1 (see Lemma 2 in [5]). Let $h \in C\left(R \times R^{m} \times R^{m}, R^{m}\right)$ be an $H_{m}$-function. Assume that $x(t)$ and $y(t)$ are continuous and satisfy

$$
x(t) \leq y(t), \quad t \in\left[t_{0}-\tau, t_{0}\right] .
$$

Furthermore, $x(t)$ is a solution of

$$
D^{+} x(t) \leq h(t, x(t), \bar{x}(t)), \quad t \geq t_{0},
$$

and $y(t)$ is a solution of

$$
\dot{y}(t)=h(t, y(t), \bar{y}(t)), \quad t \geq t_{0} .
$$

Then for all $t \geq t_{0}$,

$$
x(t) \leq y(t) .
$$

Especially, when $\tau=0$, we can get the following corollary.

Corollary 4.1 (Lemma 8.2 in [19, P.72]). Let $h \in C(R \times R, R)$. Assume that $x(t)$ and $y(t)$ are continuous. Furthermore, $x(t)$ is a solution of

$$
D^{+} x(t) \leq h(t, x(t)), \quad t \geq t_{0}
$$

and $y(t)$ is the maximal solution of

$$
\dot{y}(t)=h(t, y(t)), \quad t \geq t_{0} .
$$

Then for all $t \geq t_{0}$,

$$
x(t) \leq y(t),
$$

provided that $x\left(t_{0}\right) \leq y\left(t_{0}\right)$. 
By using the Lemma 4 in [5] and Corollary 4.1, we can easily get the following lemma.

Lemma 4.2. Let $a>0$ and $U(t, y(t))$ be continuous and nonnegative for $t_{0} \leq t<t_{0}+a, y \geq 0$. Assume that $u(t) \in C\left(\left[t_{0}-\tau, t_{0}+a\right), R\right)$ satisfies

$$
D^{+} u(t) \leq U(t, \bar{u}(t)), \quad t \in\left[t_{0}, t_{0}+a\right) .
$$

If $y(t), t \in\left[t_{0}, t_{0}+a\right)$ is the maximal solution of

$$
\dot{y}(t)=U(t, y(t)),
$$

then for all $t_{0} \leq t<t_{0}+a$,

$$
u(t) \leq y(t)
$$

provided that $\bar{u}\left(t_{0}\right) \leq y\left(t_{0}\right)$.

Theorem 4.1. Let $R_{+}=[0,+\infty)$. Assume that there is a region $G \subset\left[t_{0},+\infty\right) \times L^{p}(\Omega, C)$ such that $f$ and $g$ are continuous in $G$ and for any $(t, \phi),(t, \psi) \in G$,

$$
|f(t, \phi)-f(t, \psi)|_{\Omega}^{p} \vee|g(t, \phi)-g(t, \psi)|_{\Omega}^{p} \leq F\left(t,\|\phi-\psi\|_{\Omega}^{p}\right),
$$

where $F(t, u) \in C\left(\left[t_{0},+\infty\right) \times R_{+}, R_{+}\right)$is monotone nondecreasing with respect $u$. If for every $t_{0} \leq t<$ $+\infty, u \equiv 0$ is the only solution of the following differential equation with zero initial data

$$
\dot{u}(t)=F(t, u(t))
$$

then Eq. (1) has a unique local solution through any points in $G$.

Proof. If the conclusion of Theorem 4.1 is not true. Then there must be a point $\left(t^{*}, \xi^{*}\right) \in G$ such that there are two solutions $x(t)$ and $y(t)$ of Eq. (1) through $\left(t^{*}, \xi^{*}\right)$ with

$$
x(t) \not \equiv y(t), \quad t \in\left[t^{*}, t^{*}+a\right] \text {, for some } a>0 \text {, almost surely. }
$$

Denoting $z(t)=x(t)-y(t)$, we have

$$
z(t)=\int_{t^{*}}^{t}\left(f\left(s, x_{s}\right)-f\left(s, y_{s}\right)\right) \mathrm{d} s+\int_{t^{*}}^{t}\left(g\left(s, x_{s}\right)-g\left(s, y_{s}\right)\right) \mathrm{d} \omega(s) .
$$

Then, by Hölder's inequality and Burkholder-Davis-Gundy's inequality, we find

$$
\begin{aligned}
\|z\|_{\Omega\left[t^{*}, t\right]}^{p} \leq & 2^{p-1} \mathbb{E} \sup _{t^{*} \leq r \leq t}\left|\int_{t^{*}}^{r}\left(f\left(s, x_{s}\right)-f\left(s, y_{s}\right)\right) \mathrm{d} s\right|^{p} \\
& +2^{p-1} \mathbb{E} \sup _{t^{*} \leq r \leq t}\left|\int_{t^{*}}^{r}\left(g\left(s, x_{s}\right)-g\left(s, y_{s}\right)\right) \mathrm{d} \omega(s)\right|^{p} \\
\leq & (2 a)^{p-1} \mathbb{E} \int_{t^{*}}^{t}\left|f\left(s, x_{s}\right)-f\left(s, y_{s}\right)\right|^{p} \mathrm{~d} s \\
& +2^{p-1} a^{\frac{p-2}{2}} c_{p} \mathbb{E} \int_{t^{*}}^{t}\left|g\left(s, x_{s}\right)-g\left(s, y_{s}\right)\right|^{p} \mathrm{~d} s \\
\leq & K \int_{t^{*}}^{t} F\left(s,\left\|z_{s}\right\|_{\Omega}^{p}\right) \mathrm{d} s \\
= & K \int_{t^{*}}^{t} F\left(s, \mathbb{E} \sup _{t^{*} \leq \varrho \leq s}|z(\varrho)|^{p}\right) \mathrm{d} s \\
= & K \int_{t^{*}}^{t} F\left(s,\|z(\varrho)\|_{\Omega\left[t^{*}, s\right]}^{p}\right) \mathrm{d} s
\end{aligned}
$$


where $c_{p}=\left(p^{p+1} / 2(p-1)^{p-1}\right)^{\frac{p}{2}}$ for $p \geq 2$, and $K=(2 a)^{p-1}+2^{p-1} a^{\frac{p-2}{2}} c_{p}$.

Define $v(t)=\|z(s)\|_{\Omega\left[t^{*}, t\right]}^{p}$ and

$$
w(t)=K \int_{t^{*}}^{t} F\left(s,\|z(\varrho)\|_{\Omega\left[t^{*}, s\right]}^{p}\right) \mathrm{d} s .
$$

Since $F(t, u)$ is monotone nondecreasing with respect $u,(25)$ yields that $v(t) \leq w(t)$ and

$$
\left\{\begin{array}{l}
w^{\prime}(t)=K F(t, v(t)) \leq K F(t, w(t)), \\
w\left(t^{*}\right)=0 .
\end{array}\right.
$$

For every $t^{*} \leq t \leq t^{*}+a$, Corollary 4.1 shows that

$$
v(t) \leq w(t) \leq r(t), \quad t^{*} \leq t \leq t^{*}+a,
$$

where $r(t)$ is the maximal solution of the equation

$$
\left\{\begin{array}{l}
u^{\prime}(t)=K F(t, u(t)) \\
u\left(t^{*}\right)=0
\end{array}\right.
$$

By assumption, however, we have $r(t) \equiv 0$, and hence

$$
z(t) \equiv 0, \quad t^{*} \leq t \leq t^{*}+a, \text { almost surely, }
$$

which contradicts (23). So Theorem 4.1 is true.

\section{Remark 4.1.}

(i) If $F(t, u)$ is concave with respect to $u$, inequality (21) can be replaced by

$$
\left|f\left(t, x_{t}\right)-f\left(t, y_{t}\right)\right|^{p} \vee\left|g\left(t, x_{t}\right)-g\left(t, y_{t}\right)\right|^{p} \leq F\left(t,\|\phi-\psi\|^{p}\right) .
$$

In fact, Taking the expectation on both side of (28) and noticing that

$$
\mathbb{E} F\left(t,\|\phi-\psi\|^{p}\right) \leq F\left(t, \mathbb{E}\|\phi-\psi\|^{p}\right)
$$

when $F(t, u)$ is concave with respect to $u$, (21) can be implied by (28).

(ii) The function $F(t, u)=\lambda(t) \kappa(u), t \in\left[t_{0},+\infty\right), u \in R^{+}$is admissible in (28) provided that $\lambda(t) \geq 0$ is continuous and $\kappa(\cdot): R_{+} \rightarrow R_{+}$is continuous, monotone nondecreasing satisfying $\kappa(0)=0$, $\kappa(u)>0$ for $u>0$ and $\int_{0^{+}} \frac{\mathrm{d} u}{\kappa(u)}=\infty$ (see [6,20,21]). In particular, if $\lambda(t)=\lambda$ (positive constant), then condition (28) implies the Osgood condition (see [7,21,22,23]); if $\lambda(t)=\lambda$ and $\kappa(u)=u$, then condition (28) implies the Lipschitz condition (see [2]).

(iii) All of our results can be applied to SFDEs with infinite delays if the space $C$ is replaced by $B C\left((-\infty, 0], R^{n}\right)$ which is the space of all bounded continuous with the norm $\|\varphi\|_{*}=\sup _{-\infty<s \leq 0}|\varphi(s)|$.

Then the uniqueness result in [7] can be implied by taking $F(t, u)=\kappa(u)$ in (28).

Now, we will consider sufficient conditions for the uniqueness of solutions to SFDE (1) by using Lyapunov functions.

Let $C^{1,2}\left(R \times R^{n}, R\right)$ denote the family of all nonnegative functions $V(t, x)$ on $R \times R^{n}$ which are twice continuously differentiable in $x$ and once in $t$. For each $V(t, x) \in C^{1,2}\left(R \times R^{n}, R\right)$, we define an operator $\mathcal{L} V$, associated with the $\operatorname{SFDE}(1)$, from $R \times R^{n}$ to $R$ by

$$
\begin{gathered}
\mathcal{L} V(t, x)=V_{t}(t, x)+V_{x}(t, x) f\left(t, x_{t}\right)+\frac{1}{2} \operatorname{trace}\left[g^{T}\left(t, x_{t}\right) V_{x x} g\left(t, x_{t}\right)\right], \\
V_{t}(t, x)=\frac{\partial V(t, x)}{\partial t}, V_{x}(t, x)=\left(\frac{\partial V(t, x)}{\partial x_{1}}, \ldots, \frac{\partial V(t, x)}{\partial x_{n}}\right), V_{x x}(t, x)=\left(\frac{\partial V^{2}(t, x)}{\partial x_{i} \partial x_{j}}\right)_{n \times n} .
\end{gathered}
$$


Theorem 4.2. Denote $R_{+}^{m} \triangleq\left\{x \in R^{m}: x \geq 0\right\}$. Let $F:\left[t_{0},+\infty\right) \times R_{+}^{m} \times R_{+}^{m} \rightarrow R_{+}^{m}$ be an $H_{m}$ function with $F(t, 0,0) \equiv 0$. For every $t_{0} \leq t<+\infty, u=\left(u_{1}, \cdots, u_{m}\right)^{T} \equiv 0$ is the only solution of the following functional differential equation with zero initial data

$$
\dot{u}(t)=F(t, u(t), \bar{u}(t)) .
$$

Assume that there exists a $V=\left(V_{1}, \cdots, V_{m}\right)^{T} \in C^{1,2}\left(\left[t_{0}-\tau,+\infty\right) \times R^{n}, R_{+}^{m}\right)$ and a region $G \subset\left[t_{0},+\infty\right) \times$ $L^{p}(\Omega, C)$ such that, for any $(t, z(t)) \in G, \mathbb{E} V(t, z(t))=0$ if and only if $\mathbb{E} z(t)=0$ and

$$
\mathbb{E} \mathcal{L} V(t, z(t)) \leq F(t, \mathbb{E} V(t, z(t)), \overline{\mathbb{E} V}(t, z(t))),
$$

where $\mathcal{L} V=\left(\mathcal{L} V_{1}, \ldots, \mathcal{L} V_{m}\right)^{T}, \mathbb{E} V=\left(\mathbb{E} V_{1}, \ldots, \mathbb{E} V_{m}\right)^{T}$. Then Eq. (1) has at most one local solution through any points in $G$.

Proof. If the conclusion of Theorem 4.2 is not true. Then there must be a point $\left(t^{*}, \xi^{*}\right) \in G$ such that there are two solutions $x(t)$ and $y(t)$ of Eq. (1) through $\left(t^{*}, \xi^{*}\right)$ with $(23)$ holding. Denoting $z(t)=x(t)-y(t)$, we have

$$
z(t)=\int_{t^{*}}^{t}\left(f\left(s, x_{s}\right)-f\left(s, y_{s}\right)\right) \mathrm{d} s+\int_{t^{*}}^{t}\left(g\left(s, x_{s}\right)-g\left(s, y_{s}\right)\right) \mathrm{d} \omega(s) .
$$

By Itô's formula, we get

$$
\begin{aligned}
V(t, z(t))= & V\left(t^{*}, z\left(t^{*}\right)\right)+\int_{t^{*}}^{t} \mathcal{L} V(s, z(s)) \mathrm{d} s \\
& +\int_{t^{*}}^{t} V_{z}(s, z(s)) \cdot\left[g\left(s, x_{s}\right)-g\left(s, y_{s}\right)\right] \mathrm{d} \omega(s), \quad t \in\left[t^{*}, t^{*}+a\right] .
\end{aligned}
$$

From (30) and (32), for small enough $\Delta t>0$, we can find

$$
\begin{aligned}
\Delta \mathbb{E} V(t) & \triangleq \mathbb{E} V((t+\Delta t), z(t+\Delta t))-\mathbb{E} V(t, z(t)) \\
& =\mathbb{E} \int_{t}^{t+\Delta t} \mathcal{L} V(s, z(s)) \mathrm{d} s \\
& \leq \int_{t}^{t+\Delta t} F(s, \mathbb{E} V(s, z(s)), \overline{\mathbb{E} V}(s, z(s))) \mathrm{d} s, \quad t \in\left[t_{0}, t_{0}+a\right] .
\end{aligned}
$$

Noting $\Delta t>0$, we have

$$
\frac{\Delta \mathbb{E} V(t)}{\Delta t} \leq \frac{1}{\Delta t} \int_{t}^{t+\Delta t} F(s, \mathbb{E} V(s, z(s)), \overline{\mathbb{E} V}(s, z(s))) \mathrm{d} s, \quad t \in\left[t^{*}, t^{*}+a\right]
$$

Letting $\Delta t \rightarrow 0^{+}$, we get

$$
D^{+} \mathbb{E} V(t, z(t)) \leq F(t, \mathbb{E} V(t, z(t)), \overline{\mathbb{E} V}(t, z(t))),
$$

for all $t \in\left[t^{*}, t^{*}+a\right]$.

Since (29) has a unique solution $u \equiv 0$, then Lemma 4.1 shows that

$$
\mathbb{E} V(t, z(t)) \leq 0, \quad t^{*} \leq t \leq t^{*}+a,
$$

and the assumptions on $V$ imply that $z(t) \equiv 0$ for $t^{*} \leq t \leq t^{*}+a$ almost surely. This contradicts (23). The uniqueness has been proved.

Theorem 4.3. Let $F \in C\left(\left[t_{0},+\infty\right) \times R_{+}, R_{+}\right)$with $F(t, 0) \equiv 0$. For every $t_{0} \leq t<+\infty, u=$ $\left(u_{1}, \cdots, u_{m}\right)^{T} \equiv 0$ is the only solution of the following functional differential equation with zero initial data

$$
\dot{u}(t)=F(t, u(t))
$$


Assume that there exists a $V=\left(V_{1}, \cdots, V_{m}\right)^{T} \in C^{1,2}\left(\left[t_{0}-\tau,+\infty\right) \times R^{n}, R_{+}\right)$and a region $G \subset\left[t_{0},+\infty\right) \times$ $L^{p}(\Omega, C)$ such that, for any $(t, z(t)) \in G, \mathbb{E} V(t, z(t))=0$ if and only if $\mathbb{E} z(t)=0$ and

$$
\mathbb{E} \mathcal{L} V(t, z(t)) \leq F(t, \overline{\mathbb{E} V}(t, z(t))) .
$$

Then Eq. (1) has at most one local solution through any point in $G$.

Proof. If the conclusion of Theorem 4.3 is not true. Then there must be a point $\left(t^{*}, \xi^{*}\right) \in G$ such that there are two solutions $x(t)$ and $y(t)$ of Eq. (1) through $\left(t^{*}, \xi^{*}\right)$ with (23) holding. Denote $z(t)=x(t)-y(t)$. Using the same method in (33), we obtain

$$
D^{+} \mathbb{E} V(t, z(t)) \leq F(t, \overline{\mathbb{E} V}(t, z(t))), \quad t \in\left[t^{*}, t^{*}+a\right] .
$$

By Lemma 4.2, $\mathbb{E} V(t, z(t))$ is less than or equal to the maximal solution of (34). Therefore, we have

$$
\mathbb{E} V(t, z(t)) \leq 0, \quad t \in\left[t^{*}, t^{*}+a\right]
$$

The remainder of proof is the same with the one in Theorem 4.2.

Corollary 4.2. Suppose that all conditions of Theorem 4.3 are satisfied except that the inequality (35) is replaced by the following inequality

$$
\mathcal{L} V(t, z(t)) \leq F(t, \bar{V}(t, z(t)))
$$

Moreover, assume that $F(t, u)$ is concave with respect to $u \in R_{+}$. Then the same conclusion of Theorem 4.3 holds.

Proof. Since $F(t, u)$ is concave with respect to $u$, the inequality (36) implies that the inequality (35) holds. So, the conclusion of Corollary 4.2 is true by Theorem 4.3 .

In particular, for any $(t, \phi),(t, \psi) \in G \subset\left[t_{0}, T\right) \times L^{2}(\Omega, C)$, if we take $V(t, z(t))=|\phi-\psi|^{2}$ in Corollary 4.2 , we have the following result.

Corollary 4.3. Suppose that for any $(t, \phi),(t, \psi) \in G$,

$$
2(\phi-\psi)^{T}(f(t, \phi)-f(t, \psi))+|g(t, \phi)-g(t, \psi)|^{2} \leq F\left(t,\|\phi-\psi\|^{2}\right),
$$

where $F(t, u)$ satisfies the conditions in Corollary 4.2. Then the same conclusion of Theorem 4.3 holds.

Remark 4.2. Fedorenko [24] studied the uniqueness of Eq. (1) without delays. The main results in [24] can be implied by Corollary 4.3.

\section{Continuation and Global Existence Theorems}

In this section, we will give some continuation and global existence theorems for SFDE (1) under nonLipschitz condition with Lipschitz condition being considered as a special case.

Theorem 5.1. Assume that $f$ and $g$ are continuous on $\left[t_{0}, T\right) \times L^{p}(\Omega, C)$, and the following conclusions are true.

(I) The initial value problem (1) has a non-continuable solution $x(t)$, whose maximum existing interval is assumed to be $\left[t_{0}-\tau, \beta_{1}\right)$, where $t_{0}<\beta_{1} \leq T$.

(II) The problem (1) has a unique non-continuable solution on $\left[t_{0}-\tau, \beta_{1}\right)$ if its solution is local existence and uniqueness for any $\left(t^{*}, \xi^{*}\right) \in\left[t_{0}, \beta_{1}\right) \times L^{p}(\Omega, C)$.

(III) For any compact set $D \subset\left[t_{0}, T\right) \times L^{p}(\Omega, C)$,

$$
\left(t, x_{t}\right) \notin D, \text { for some } t \in\left[t_{0}, \beta_{1}\right) \text {. }
$$


Proof. The proof of (I) is similar to that of (I) of Theorem 2 in [5], so we omit it.

To prove (II), assume that $x_{t}$ and $\hat{x}_{t}$ are two non-continuable solutions of Eq. (1) through $\left(t^{*}, \xi^{*}\right)$. Now we will show that the two solutions have the same maximum existing interval $J \subset\left[t_{0}, \beta_{1}\right)$ and

$$
P\left\{x_{t}=\hat{x}_{t} \text { for all } t \in J\right\}=1 .
$$

If (39) is not true, we can find $t_{1}, t_{2} \in\left[t_{0}, \beta_{1}\right)$ with $t_{1}<t_{2}$ such that

$$
P\left\{x_{t_{1}}=\hat{x}_{t_{1}}\right\}=1, P\left\{x_{t}=\hat{x}_{t} \text { for all } t_{1}<t \leq t_{2}\right\}<1 \text {. }
$$

It is obvious that $\left(t_{1}, x_{t_{1}}\right) \in\left[t_{0}, \beta_{1}\right) \times L^{p}(\Omega, C)$. By the assumption of (II), there is a neighborhood $U \subset\left[t_{0}, \beta_{1}\right) \times L^{p}(\Omega, C)$ such that there is only one solution of (1) through $\left(t_{1}, x_{t_{1}}\right)$ in $U$. However, $x_{t}$ and $\hat{x}_{t}$ are both the solution of Eq. (1) through $\left(t_{1}, x_{t_{1}}\right)$. For small enough $\epsilon>0$, when $0<\left|t-t_{1}\right| \leq \epsilon$, we have

$$
P\left\{\left(t, x_{t}\right) \in U\right\}=1, \quad P\left\{\left(t, \hat{x}_{t}\right) \in U\right\}=1,
$$

and

$$
P\left\{x_{t}=\hat{x}_{t} \text { for all } 0<\left|t-t_{1}\right| \leq \epsilon\right\}=1,
$$

which contradicts the inequality of (40). Thus (II) holds.

In (III), the case $\beta_{1}=T$ is trivial. So we suppose $\beta_{1}<T$. If the conclusion of (III) is not true, there are a compact set $D \subset\left[t_{0}, T\right) \times L^{p}(\Omega, C)$ and a sequence of real numbers $t_{k} \rightarrow \beta_{1}^{-}$as $k \rightarrow+\infty$, and a $\psi \in L^{p}(\Omega, C)$ such that

$$
\left(t_{k}, x_{t_{k}}\right) \in D, \quad\left(\beta_{1}, \psi\right) \in D, \quad\left(t_{k}, x_{t_{k}}\right) \rightarrow\left(\beta_{1}, \psi\right), \text { as } k \rightarrow+\infty
$$

Thus, for any $\varepsilon>0$,

$$
\lim _{k \rightarrow+\infty} \mathbb{E} \sup _{\theta \in[-\tau,-\varepsilon]}\left|x_{t_{k}}(\theta)-\psi(\theta)\right|^{p}=0 .
$$

Since $x_{t}(\theta)=x(t+\theta),-\tau \leq \theta \leq 0$, and $\tau>0$, this implies

$$
\mathbb{E}\left|x\left(\beta_{1}+\theta\right)-\psi(\theta)\right|^{p}=0, \quad-\tau \leq \theta<0 .
$$

Hence $\lim _{t \rightarrow \beta_{1}^{-}} x(t)$ exists, and $x(t)$ can be continued to $\left[t_{0}-\tau, \beta_{1}\right]$. This contradicts the fact that the maximum existing interval of $x(t)$ is $\left[t_{0}-\tau, \beta_{1}\right)$. So, (III) is true.

Remark 5.1. Combining the uniqueness theorems established in section 4, the conclusion (II) of Theorem 5.1 implies that a unique non-continuable solution of SFDE (1) always exists under the conditions of theorems in Section 4 .

Theorem 5.2. Assume that $f$ and $g$ are continuous on $\left[t_{0}, T\right) \times L^{p}(\Omega, C)$ and quasi-bounded in $\left[t_{0}, T\right) \times$ $L^{p}(\Omega, C)$. If $x(t)$ is a non-continuable solution of (1) on $\left[t_{0}-\tau, \beta_{1}\right)$. Then, for every closed bounded set $A \subset\left[t_{0}-\tau, T\right) \times L^{p}(\Omega, C)$,

$$
(t, x(t)) \notin A, \text { for some } t \in\left[t_{0}, T\right) .
$$

Proof. The case $\beta_{1}=T$ is trivial. So we suppose $\beta_{1}<T$. If the conclusion of the theorem is not true, there must exist a closed bounded set $A \subset\left[t_{0}-\tau, T\right) \times L^{p}(\Omega, C)$ such that

$$
\left(t, x_{t}\right) \in A, \text { for all } t \in\left[t_{0}, \beta_{1}\right) \text {. }
$$

From the boundedness of $A$, there exists a constant $\alpha_{1}>\|\xi\|_{\Omega}$ such that

$$
\left\|x_{t}\right\|_{\Omega}<\alpha_{1}, \text { for all } t \in\left[t_{0}, \beta_{1}\right)
$$

By the quasi-boundedness of $f$ and $g$, there is a positive constant $\mu$ such that

$$
\left|f\left(t, x_{t}\right)\right|_{\Omega} \leq \mu, \quad\left|g\left(t, x_{t}\right)\right|_{\Omega} \leq \mu, \text { for all } t \in\left[t_{0}, \beta_{1}\right) .
$$


By using the properties of Brownian motion and (44), we obtain for all $t_{1}, t_{2} \in\left[t_{0}, \beta_{1}\right)$,

$$
\begin{aligned}
\left|x\left(t_{1}\right)-x\left(t_{2}\right)\right|_{\Omega} & \leq 2\left|\int_{t_{1}}^{t_{2}} f\left(s, x_{s}\right) \mathrm{d} s\right|_{\Omega}+2\left|\int_{t_{1}}^{t_{2}} g\left(s, x_{s}\right) \mathrm{d} \omega(s)\right|_{\Omega} \\
& \leq 2 \mu\left|t_{2}-t_{1}\right|+2 \mu C_{p}^{\frac{1}{p}}\left|t_{2}-t_{1}\right|^{\frac{1}{2}} .
\end{aligned}
$$

This implies that $\left\{\left(t, x_{t}\right): t_{0} \leq t<\beta_{1}\right\}$ belongs to a compact set in $\left[t_{0}, T\right) \times L^{p}(\Omega, C)$. This contradicts Theorem 5.1 and we prove the theorem.

The following corollary is easy implied by Theorem 5.2 .

Corollary 5.1. With the same conditions in Theorem 5.2, the following conclusions are true.

(I) If $\beta_{1}<T$, then the solution $x(t)$ of the SFDE (1) explodes in $\left[t_{0}-\tau, \beta_{1}\right)$.

(II) If the solution $x(t)$ of (1) is bounded, then $x(t)$ exists on $\left[t_{0}-\tau, T\right)$.

Theorem 5.3. Suppose that $f$ and $g$ are quasi-bounded, continuous on $\left[t_{0}, T\right) \times L^{p}(\Omega, C)$, and there exists a function $F(t, u) \in C\left(\left[t_{0}, T\right) \times R_{+}, R_{+}\right)$such that for all $t \in\left[t_{0}, T\right)$ and $\phi \in L^{p}(\Omega, C)$

$$
|f(t, \phi)|^{p}+|g(t, \phi)|^{p} \leq F\left(t,\|\phi\|^{p}\right),
$$

where $F(t, u)$ is monotone nondecreasing and concave with respect to $u \in R_{+}$for each fixed $t \in\left[t_{0}, T\right)$. If for any $\gamma>0$ and arbitrary given initial condition, the solution of the scalar differential equation

$$
u^{\prime}(t)=\gamma F(t, u)
$$

exists on $\left[t_{0}, T\right)$. Then any solution of (1) exists also on $\left[t_{0}-\tau, T\right)$.

Proof. From Theorem 5.1, the SFDE (1) has a solution $x(t)=x\left(t ; t_{0}, \xi\right)$ with maximum existing interval $\left[t_{0}-\tau, \beta_{1}\right)$. Now, we only need to prove $\beta_{1}=T$. If $\beta_{1}<T$, by Corollary 5.1 , there exists a measurable subset $S \subset \Omega$ with $P(S)>0$ such that $x(t)$ explodes in $\left[t_{0}-\tau, \beta_{1}\right)$ for all $\omega \in S$. For any sufficiently large integer $n$, we define the stopping times

$$
\tau_{n}=\beta_{1} \wedge \inf \left\{t \in\left[t_{0}, \beta_{1}\right):|x(t)| \geq n\right\},
$$

where, as usual, we set $\inf \emptyset=\infty$. Clearly, $\tau_{n}$ 's are increasing. So they have the $\operatorname{limit} \beta_{1}=\lim _{n \rightarrow \infty} \tau_{n}$. Since

$$
x\left(t \wedge \tau_{n}\right)=\xi(0)+\int_{t_{0}}^{t \wedge \tau_{n}} f\left(s, x_{s}\right) \mathrm{d} s+\int_{t_{0}}^{t \wedge \tau_{n}} g\left(s, x_{s}\right) \mathrm{d} \omega(s), \quad t \in\left[t_{0}, \beta_{1}\right) .
$$

By the assumptions, we obtain that

$$
\begin{aligned}
\mathbb{E} I_{S}\left|x\left(t \wedge \tau_{n}\right)\right|^{p} \leq & 3^{p-1} \mathbb{E} I_{S}|\xi(0)|^{p}+3^{p-1} \mathbb{E} I_{S}\left|\int_{t_{0}}^{t \wedge \tau_{n}} f\left(s, x_{s}\right) \mathrm{d} s\right|^{p} \\
& +3^{p-1} \mathbb{E} I_{S}\left|\int_{t_{0}}^{t \wedge \tau_{n}} g\left(s, x_{s}\right) \mathrm{d} \omega(s)\right|^{p} \\
\leq & 3^{p-1} \mathbb{E} I_{S}|\xi(0)|^{p}+3^{p-1}\left(\beta_{1}-t_{0}\right)^{p-1} \mathbb{E} I_{S} \int_{t_{0}}^{t \wedge \tau_{n}}\left|f\left(s, x_{s \wedge \tau_{n}}\right)\right|^{p} \mathrm{~d} s \\
& +3^{p-1} C_{p}\left(\beta_{1}-t_{0}\right)^{\frac{p-2}{2}} \mathbb{E} I_{S} \int_{t_{0}}^{t \wedge \tau_{n}}\left|g\left(s, x_{s \wedge \tau_{n}}\right)\right|^{p} \mathrm{~d} s \\
\leq & 3^{p-1} \mathbb{E} I_{S}|\xi(0)|^{p}+3^{p-1} K_{p} \mathbb{E} I_{S} \int_{t_{0}}^{t \wedge \tau_{n}} F\left(s,\left\|x_{s \wedge \tau_{n}}\right\|^{p}\right) \mathrm{d} s \\
\leq & 3^{p-1} \mathbb{E} I_{S}|\xi(0)|^{p}+3^{p-1} K_{p} \int_{t_{0}}^{t} F\left(s, \mathbb{E} I_{S}\left\|x_{s \wedge \tau_{n}}\right\|^{p}\right) \mathrm{d} s,
\end{aligned}
$$


where $K_{p}=\left(\beta_{1}-t_{0}\right)^{p-1}+C_{p}\left(\beta_{1}-t_{0}\right)^{\frac{p-2}{2}}$. Let $u(t)$ denote the maximal solution of the scalar differential equation (47) with $\gamma=3^{p-1} K_{p}$ and the initial value $u_{0} \geq 3^{p-1} \mathbb{E} I_{S}\|\xi\|^{p}$.

Similar to the proof of (25)-(27) in Theorem 4.1, we obtain that

$$
\mathbb{E} I_{S}\left|x\left(t \wedge \tau_{n}\right)\right|^{p} \leq u\left(t \wedge \tau_{n}\right), \quad t \in\left[t_{0}, \beta_{1}\right), \quad \text { for each } n \gg 1
$$

So,

$$
\mathbb{E} I_{S}\left|x\left(\beta_{1} \wedge \tau_{n}\right)\right|^{p} \leq u\left(\beta_{1} \wedge \tau_{n}\right), \text { for each } n \gg 1
$$

Since the solution of (47) exists in $\left[t_{0}-\tau, T\right)$, we have $u\left(\beta_{1} \wedge \tau_{n}\right)<\infty$. However, the left side of (51) approaches to $\infty$ as $n \rightarrow \infty$, which is a contradiction. Consequently, the proof is completed.

By Theorem 5.3 and Lemma 5 in [5], we have the following result.

Corollary 5.2. Suppose that $f$ and $g$ are continuous on $\left[t_{0}, T\right) \times L^{p}(\Omega, C)$ and for all $t \in\left[t_{0}, T\right)$, $\phi \in L^{p}(\Omega, C)$

$$
|f(t, \phi)|^{p}+|g(t, \phi)|^{p} \leq a(t)+b(t) k\left(\|\phi\|^{p}\right),
$$

where $a(t), b(t) \in C\left(\left[t_{0}, T\right), R_{+}\right)$and $k(u) \in C\left(R_{+}, R_{+}\right)$is monotone nondecreasing, concave and satisfies

$$
\int_{0}^{+\infty} \frac{\mathrm{d} u}{1+k(u)}=+\infty .
$$

Then any solution of (1) exists on $\left[t_{0}-\tau, T\right)$.

The following theorems on global existence of the solution of (1) can be implied by the analogous methods in [5].

Theorem 5.4. Let the conditions of Theorem 5.2 hold. Suppose that there are functions $V \in C^{1,2}\left(\left[t_{0}-\right.\right.$ $\left.\tau, T) \times R^{n}, R_{+}^{m}\right)$ and $F \in C\left(\left[t_{0}, T\right) \times R_{+}^{m} \times R_{+}^{m}, R_{+}^{m}\right)$ such that

$$
\begin{aligned}
& \max _{1 \leq i \leq m}\left\{\lim _{|x| \rightarrow \infty}\left[\inf _{t_{0}-\tau \leq t<T} V_{i}(t, x)\right]\right\}=\infty, \\
& \mathbb{E} \mathcal{L} V(t, x) \leq F(t, \mathbb{E} V(t, x), \overline{\mathbb{E} V}(t, x)), \quad \forall t \in\left[t_{0}, T\right), x \in R^{n},
\end{aligned}
$$

where $\mathcal{L} V=\left(\mathcal{L} V_{1}, \ldots, \mathcal{L} V_{m}\right)^{T}, \mathbb{E} V=\left(\mathbb{E} V_{1}, \ldots, \mathbb{E} V_{m}\right)^{T}$ and $R_{+}=[0, \infty)$.

Assume moreover that $F$ is an $H_{m}$-function and for arbitrary given initial condition, the solution $u(t)$ of the delay differential equation

$$
\dot{u}(t)=F(t, u(t), \bar{u}(t))
$$

exists on $\left[t_{0}-\tau, T\right)$. Then any solution of (1) exists also on $\left[t_{0}-\tau, T\right)$.

Theorem 5.5. Let the conditions of Theorem 5.2 hold. Suppose that there are functions $V \in C^{1,2}\left(\left[t_{0}-\right.\right.$ $\left.\tau, T) \times R^{n}, R_{+}\right)$and $F \in C\left(\left[t_{0}, T\right) \times R_{+}, R_{+}\right)$such that

$$
\begin{aligned}
& \lim _{|x| \rightarrow \infty}\left[\inf _{t_{0}-\tau \leq t<T} V(t, x)\right]=\infty, \\
& \mathbb{E} \mathcal{L} V(t, x) \leq F(t, \overline{\mathbb{E} V}(t, x)), \quad \forall t \in\left[t_{0}, T\right), x \in R^{n} .
\end{aligned}
$$

Assume moreover that for arbitrary given initial condition, the maximal solution $u(t)$ of the differential equation

$$
\dot{u}(t)=F(t, u(t))
$$

exists on $\left[t_{0}, T\right)$. Then any solution of (1) exists also on $\left[t_{0}-\tau, T\right)$. 


\section{Example}

In this section we present an example to illustrate our results obtained above. For this, we need the following lemma which can be implied by the definition of concave function (see Kuang [25, P.369-370]).

Lemma 6.1. Suppose that $f(x): D \subset R \rightarrow R$ is a concave function. Then, for any $x_{i} \in D(i=1, \cdots, 4)$ with $x_{1} \leq x_{2}<x_{3} \leq x_{4}$, we have

$$
\frac{f\left(x_{3}\right)-f\left(x_{1}\right)}{x_{3}-x_{1}} \geq \frac{f\left(x_{4}\right)-f\left(x_{2}\right)}{x_{4}-x_{2}}
$$

Furthermore, if $x_{1}<x_{2} \leq x_{3}<x_{4}$, we get

$$
\frac{f\left(x_{2}\right)-f\left(x_{1}\right)}{x_{2}-x_{1}} \geq \frac{f\left(x_{4}\right)-f\left(x_{3}\right)}{x_{4}-x_{3}} .
$$

Example 6.1. Consider the following SFDE:

$$
\mathrm{d} x(t)=f\left(t, x_{t}\right) \mathrm{d} t+g\left(t, x_{t}\right) \mathrm{d} \omega(t), \quad t_{0} \leq t<+\infty,
$$

with the initial condition

$$
x_{t_{0}}=\xi \in L^{2}(\Omega, C([-\tau, 0], R)), \quad s \in[-\tau, 0],
$$

In (60) $f\left(t, x_{t}\right)=a(t) f_{0}\left(x_{t}\right)$ and $g\left(t, x_{t}\right)=b(t)\left(\sin x_{t}-1\right)$, where $a(t), b(t)$ are continuous functions on $\left[t_{0},+\infty\right)$, and

$$
f_{0}(s)= \begin{cases}0, & s=0 \\ s \ln ^{\frac{1}{2}} s^{-2}, & 0<s \leq e^{-2} \\ 2 e^{-1} \sqrt{s}, & s>e^{-2}\end{cases}
$$

Then $f\left(t, x_{t}\right)$ and $g\left(t, x_{t}\right)$ are continuous on $\left[t_{0},+\infty\right) \times L^{2}(\Omega, C([-\tau, 0], R))$. It follows from Theorem 3.1 that SFDE (60) and (61) has a local solution $x_{t} \in L^{2}(\Omega, C([-\tau, 0], R))$.

We note that for $s>0$

$$
\frac{\mathrm{d} f_{0}(s)}{\mathrm{d} s}= \begin{cases}\frac{-2 \ln s-1}{\sqrt{-2 \ln s}}>0, & 0<s<e^{-2}, \\ e^{-1} s^{-\frac{1}{2}}>0, & s>e^{-2},\end{cases}
$$

and

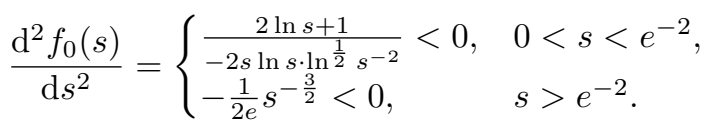

Moreover, $f_{0}^{\prime}\left(e^{-2}-0\right)=\frac{3}{2}>f_{0}^{\prime}\left(e^{-2}+0\right)=1$. Thus we can find that $f_{0}$ is monotone increasing and concave for any $s \geq 0$.

To discuss the uniqueness, we will estimate $\left|f_{0}\left(s_{2}\right)-f_{0}\left(s_{1}\right)\right|$ for $s_{1}, s_{2} \in R_{+}$. Without loss of generality, let $0<s_{1}<s_{2}$. Then there are only two cases: $s_{2}-s_{1} \leq s_{1}$ or $s_{2}-s_{1}>s_{1}$. That is, $0<s_{1}<s_{2}-s_{1}<s_{2}$ or $0<s_{2}-s_{1} \leq s_{1}<s_{2}$.

For both of the cases, by using the concavity of $f_{0}$ on $[0, \infty)$, it follows from (58) and (59) in Lemma 6.1 that

$$
\frac{f_{0}\left(s_{2}-s_{1}\right)-f_{0}(0)}{s_{2}-s_{1}} \geq \frac{f_{0}\left(s_{2}\right)-f_{0}\left(s_{1}\right)}{s_{2}-s_{1}}
$$

that is

$$
f_{0}\left(s_{2}\right)-f_{0}\left(s_{1}\right) \leq f_{0}\left(s_{2}-s_{1}\right)
$$

Then

$$
\left|f\left(t, s_{1}\right)-f\left(t, s_{2}\right)\right| \leq|a(t)| f_{0}\left(s_{2}-s_{1}\right), \quad \forall s_{1}, s_{2} \in R_{+} .
$$


We choose that

$$
h(s)= \begin{cases}0, & s=0, \\ -s \ln s, & 0<s \leq e^{-4} \\ 4 e^{-2} s^{\frac{1}{2}}, & s>e^{-4}\end{cases}
$$

Clearly, $h(s)$ is continuous, monotone increasing and concave for all $s \in R_{+}$.

For any $\varphi_{1}, \varphi_{2} \in L^{2}(\Omega, C([-\tau, 0], R))$, it follows from (64) that

$$
\left|f\left(t, \varphi_{1}\right)-f\left(t, \varphi_{2}\right)\right|^{2} \leq a^{2}(t) h\left(\left\|\varphi_{1}-\varphi_{2}\right\|^{2}\right), \quad \forall t \geq t_{0} .
$$

On the other hand, $g\left(t, x_{t}\right)$ satisfies

$$
\left|g\left(t, \varphi_{1}\right)-g\left(t, \varphi_{2}\right)\right| \leq b(t)\left\|\varphi_{1}-\varphi_{2}\right\|, \quad \forall t \geq t_{0} .
$$

Let $\lambda(t)=\max \left\{a^{2}(t), b^{2}(t)\right\}$ and $F(t, u)=\lambda(t) \kappa(u)$ where $\kappa(s)=h(s)+s$. Then $\lambda(t)$ is a continuous function on $\left[t_{0},+\infty\right)$ and $\kappa(s)$ is also continuous, monotone nondecreasing and concave for all $s \in R_{+}$. Thus, it follows from (65) and (66) that for any $\varphi_{1}, \varphi_{2} \in L^{2}(\Omega, C([-\tau, 0], R))$ and $t \geq t_{0}$

$$
\begin{aligned}
\left|f\left(t, \varphi_{1}\right)-f\left(t, \varphi_{2}\right)\right|^{2}+\left|g\left(t, \varphi_{1}\right)-g\left(t, \varphi_{2}\right)\right|^{2} & \leq a^{2}(t) h\left(\left\|\varphi_{1}-\varphi_{2}\right\|^{2}\right)+b^{2}(t)\left\|\varphi_{1}-\varphi_{2}\right\|^{2} \\
& \leq \lambda(t)\left(h\left(\left\|\varphi_{1}-\varphi_{2}\right\|^{2}\right)+\left\|\varphi_{1}-\varphi_{2}\right\|^{2}\right) \\
& =\lambda(t) \kappa\left(\left\|\varphi_{1}-\varphi_{2}\right\|^{2}\right) .
\end{aligned}
$$

Moreover, we have

$$
\int_{0^{+}} \frac{\mathrm{d} s}{\kappa(s)}=\int_{0^{+}} \frac{\mathrm{d} s}{-s \ln s+s}=-\left.\ln \ln \frac{e}{s}\right|_{0^{+}}=+\infty
$$

It follows from (67) and Theorem 4.1 that the local solution $x_{t}$ of SFDE (60) and (61) is unique for any $\left(t_{0}, \xi\right) \in R \times L^{2}(\Omega, C([-\tau, 0], R))$. Then Theorem 5.1 implies that $S F D E(60)$ and (61) has a unique non-continuable solution.

On the other hand, for any $\varphi \in L^{2}(\Omega, C([-\tau, 0], R))$, we get

$$
\begin{aligned}
|f(t, \varphi)|^{2}+|g(t, \varphi)|^{2} \leq & 2\left(|f(t, \varphi)-f(t, 0)|^{2}+|f(t, 0)|^{2}\right) \\
& +2\left(|g(t, \varphi)-g(t, 0)|^{2}+|g(t, 0)|^{2}\right) \\
\leq & 2 a^{2}(t) h\left(\|\varphi\|^{2}\right)+2 b^{2}(t)\|\varphi\|^{2}+2\left(|f(t, 0)|^{2}+|g(t, 0)|^{2}\right) \\
\leq & 2 \lambda(t) \kappa\left(\|\varphi\|^{2}\right)+2 b^{2}(t) .
\end{aligned}
$$

We can compute that

$$
\begin{aligned}
\int_{0}^{+\infty} \frac{\mathrm{d} s}{1+\kappa(s)} & =\int_{0^{+}}^{e^{-4}} \frac{\mathrm{d} s}{-s \ln s+s+1}+\int_{e^{-4}}^{+\infty} \frac{\mathrm{d} s}{4 e^{-4} \sqrt{s}+s+1} \\
& \geq \int_{e^{-4}}^{+\infty} \frac{\mathrm{d} s}{4 e^{-4} \sqrt{s}+s+1}=+\infty
\end{aligned}
$$

Then it follows from Corollary 5.2 that the unique non-continuable solution of (60) and (61) is also global existence.

Remark 6.1. Since $f$ does not satisfy the Lipschitz condition, the results obtained in [5] are not applicable to SFDE (60) and (61).

\section{Conclusion}

This paper is concerned with some basic theories of solutions for SFDEs with non-Lipschitz coefficients. Firstly, we obtain the Peano's theorem for SFDEs. Our new theorems need only the continuity condition to prove a local existence theorem for SFDEs. Then, some new uniqueness theorems are established by the comparison methods. Finally, we give some continuation theorems and global existence theorems for SFDEs with non-Lipschitz coefficients. The gained results generalize and improve some of the existing results mentioned in the literature. An example is presented to illustrate the efficiency of the obtained results. 
Acknowledgments. The authors would like to thank the reviewers for their constructive suggestions and helpful comments. This work was supported by the Fundamental Research Funds for the Central Universities, Southwest University for Nationalities (Grant No. 13NZYBS07) and Scientific Reserch Fund of SiChuan Provincial Education Department (Grant No. 15ZB0492).

\section{References}

1. L. Arnold, Stochastic Differential Equations: Theory and Applications, Wiley, New York, 1972.

2. S-E A Mohammed, Stochastic Functional Differential Equations, Pitman Publishing Program, Boston, 1984.

3. X. Mao, Stochastic Differential Equations and Applications, Horwood, 1997.

4. D. Y. Xu, Y. M. Huang and Z. G. Yang, "Existence theorems for periodic Markov process and stochastic functional differential equations," Discrete Contin. Dyn. Syst. vol.24,no.3, pp. 1005-1023, 2009.

5. D. Y. Xu, Z. G. Yang and Y. M. Huang, "Existence-uniqueness and continuation theorems for stochastic functional differential equations," J. Differential Equations, vol.245, pp. 1681-1703, 2008.

6. J. Turo, "Successive approximations of solutions to stochastic functional differential equations," Period. Math. Hungar. vol.30, no.1, pp. 87-96, 1995.

7. Y. Ren, S. Lu and N. Xia, "Remarks on the existence and uniqueness of the solutions to stochastic functional differential equations with infinite delay," J. Comput. Appl. Math. vol.220, no.1-2, pp. 364-372, 2008.

8. D. Y. Xu, B. Li, S.J. Long and L.Y. Teng, "Moment estimate and existence for solutions of stochastic functional differential equations," Nonlinear Anal. vol.108, pp. 128-143, 2014.

9. P. Hartman, Ordinary Differential Equations, Birkhauser, Boston 1982.

10. I. Hirai and K. Ako, "On generalized Peano's theorem concerning the Dirichlet problem for semi-linear elliptic differential equations," Proc. Japan Academy, vol.36, pp. 480-485, 1960.

11. J. K. Hale and S. M. V. Lunel, Introduction to Functional Differential Equations, Springer-Verlag, New York, 1993.

12. J-P. Aubin and H, Frankowska, Set-Valued Analysis, Birkhauser, Basel, 1991.

13. O. Kaleva, "The Cauchy problem for fuzzy differential equations," Fuzzy Sets and Systems, vol.35, pp. 389-396, 1990.

14. P. E. Kloeden, "Remarks on Peano-like theorems for fuzzy differential equations," Fuzzy Sets and Systems, vol.44, pp. 161-163, 1991.

15. Juan J. Nieto, "The Cauchy problem for continuous fuzzy differential equations," Fuzzy Sets and Systems, vol.102, pp. 259-262, 1999.

16. V. Lakshmikantham and A. S. Vatsala, "Basic theory of fractional differential equations," Nonlinear Anal. vol.69, pp. 2677-2682, 2008.

17. T. A. Burton, Stability and Periodic Solutions of Ordinary and Functional Differential Equations, Dover, New York, 2005.

18. E. Zeidler, Nonlinear Functional Analysis and its Applications I Fixed-Point Theorems, Springer-Verlag, New York, 1986.

19. R. K. Miller and A. N. Michel, Ordinary Differential Equations, Academic Press, New York, 1982.

20. T. Taniguchi, "On sufficient conditions for nonexplosion of solutions to stochastic differential equations," J. Math. Anal. Appl. vol.153, pp. 549-561, 1990.

21. T. Taniguchi, "Successive approximations to solutions of stochastic differential equations," J. Differential Equations, vol.96, pp. 152-169, 1992.

22. T. Yamada, "On the successive approximation of solutions of stochastic differential equations," J. Math. Sci. Univ. Kyoto, vol.21, pp. 501-515, 1981.

23. G. S. Ladde and S. Seikkala, "Existence, uniqueness and upper estimates for solutions of McShane type stochastic differential systems," Stoch. Anal. Appl. vol.4, pp. 409-429, 1986.

24. I. V. Fedorenko, "Existence and uniqueness of a continuous solution of a system of stochastic differential equations of Itô type," Russ. Math. Surv. vol.41, pp. 227-228, 1986.

25. J. C. Kuang, Applied Inequalities, 3nd edn. Shandong Science and Technology Press, 2004. 